\title{
Diagnostic genetic testing for Huntington's disease
}

\author{
David Craufurd, ${ }^{1,2}$ Rhona MacLeod, ${ }^{1,2}$ Marina Frontali, ${ }^{3}$ Oliver Quarrell, ${ }^{4}$ \\ Emilia K Bijlsma, ${ }^{5}$ Mary Davis, ${ }^{6}$ Lena Elisabeth Hjermind, ${ }^{7,8}$ \\ Nayana Lahiri, ${ }^{9}$ Paola Mandich, ${ }^{10,11}$ Asunción Martinez, ${ }^{12}$ Aad Tibben, ${ }^{5}$ \\ Raymund A Roos, ${ }^{13}$ on behalf of the Working Group on Genetic \\ Counselling and Testing of the European Huntington's Disease Network \\ (EHDN)
}

For numbered affiliations see end of article.

\section{Correspondence to}

Dr D Craufurd, Manchester Centre for Genomic Medicine, St. Mary's Hospital, Oxford Road, Manchester M13 9WL, UK; david.craufurd@manchester. ac.uk

Accepted 2 August 2014 Published Online First 28 August 2014
CrossMark

To cite: Craufurd $D_{\text {, }}$

MacLeod R, Frontali M, et al.

Pract Neurol 2015;15:80-84.

\section{INTRODUCTION}

Huntington's disease (HD) is an inherited neurodegenerative disorder characterised by a combination of motor abnormalities (chorea, dystonia, hypokinesia), cognitive impairment and neuropsychiatric symptoms, including depression, irritability and apathy. The age at onset is typically $35-45$ years but it can present in juveniles and the elderly. ${ }^{1}$ The disease slowly progresses over $15-20$ years. ${ }^{1}$ It is inherited as an autosomal dominant trait, with each child of an affected parent having a $50 \%$ risk of carrying the gene mutation and therefore of developing HD themselves. The cause is a CAG trinucleotide expansion mutation in the HTT gene that encodes the ubiquitously expressed huntingtin protein. Genetic testing by direct mutation analysis has been available clinically since the mutation was identified in $1993 .^{2}$ Measurement of the CAG repeat length acts as a trait marker rather than a state marker; that is, the genetic test can be used either to confirm a clinical diagnosis of HD in symptomatic people or to predict whether or not an at-risk person will go on to develop HD. An abnormal result indicates that the person will one day develop HD, but gives no indication of his or her clinical state at the time of testing.

The pretest counselling requirements differ for diagnostic and presymptomatic (predictive) testing. The variety and complexity of clinical situations where diagnostic genetic testing for HD may be indicated make it impractical to construct guidelines such as those governing predictive testing. ${ }^{3} 4$ The purpose of this article is to draw the attention of neurologists and psychiatrists to the challenges and pitfalls encountered in diagnostic testing for HD. We make the following recommendations to guide practice and help ensure the best possible outcome for individuals and their family.

A diagnostic genetic test is carried out where an individual is already symptomatic. According to current convention, the symptoms must include unequivocal motor signs. Neurologists (or neuropsychiatrists) are generally the professionals responsible for the diagnosis of HD. Mood disturbance, personality changes or cognitive impairment and a positive family history, without motor signs, are not sufficiently specific to justify a diagnostic genetic test. Testing in such circumstances where the patient wishes to know his or her genetic status is a predictive test, requiring referral to a genetic counselling unit.

A predictive genetic test is an option for people at risk of developing HD. It provides information about whether someone will develop symptoms in the future, and, according to internationally agreed guidelines, can only be requested by a genetic counselling unit. ${ }^{4}$ Pretest counselling is an essential requirement to allow a patient adequate opportunity to reflect on what will be an irrevocable decision.

\section{DIAGNOSTIC GENETIC TESTING}

Confirming the clinical diagnosis of an inherited disorder has consequences for the patient and other family members (figure 1). Careful attention to timing and needs of the family can maximise the potential therapeutic value of the diagnostic process. For example, a diagnosis sometimes helps to explain worrying symptoms and thus comes as a relief to 


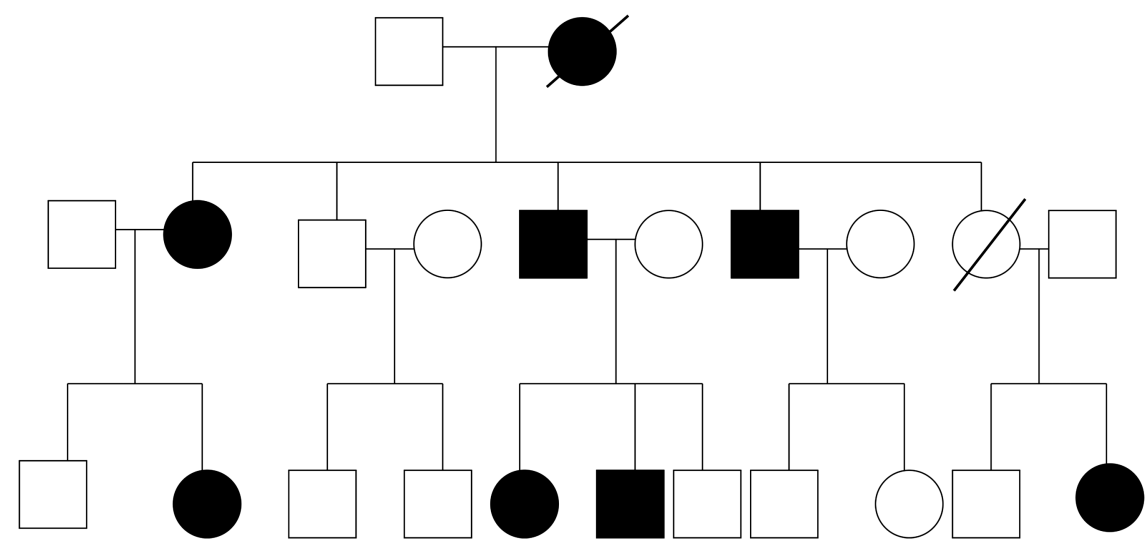

Figure 1 A typical pedigree for a family affected with Huntington's disease (HD). The autosomal dominant mode of inheritance can lead to multiple cases over many generations, so an unfavourable test result often has serious implications for many other family members.

the patient and his or her family; however, a diagnosis of HD can induce or worsen existing depression: the risk of suicide and suicidal ideation increases just before and after disease onset. ${ }^{56}$ Where a diagnosis is confirmed, the patient's partner, children, grandchildren and even siblings may need a lot of information, especially if there is no previously known family history of HD.

\section{WHEN SHOULD WE CONSIDER DIAGNOSTIC GENETIC TESTING}

The patient with a positive family history and specific motor symptoms

In this situation, the clinical diagnosis is not difficult; the patient is usually aware of symptoms, and the test becomes little more than a formality. However, even this can be more complicated than it first appears. Many patients with early HD are unaware of the involuntary movements and the early behavioural changes occurring around the time of onset. ${ }^{78}$ Some people have a striking lack of insight into their symptoms, which can persist until the disease is quite advanced. $^{9-12}$ Commonly, the patient's spouse or another family member has suspected the diagnosis for some time before the patient became aware of symptoms. In some cases, a family member may even have prompted the referral, while the patient remains convinced that he or she is perfectly well. Such a person is unprepared for the diagnosis and may not be able, in the fullest sense, to give informed consent. The entire process of imparting the diagnosis and performing a confirmatory test requires care and sensitivity to avoid causing unnecessary distress and to avoid driving the person into a state of entrenched denial, which may hinder subsequent attempts at symptomatic treatment. It is, therefore, important to explore the patient's understanding of the situation before proceeding to genetic testing; extra time spent on this can save a great deal of trouble later. An additional appointment and/or offering additional support before diagnostic testing may help.

The patient with no family history, but specific symptoms likely to be HD

Diagnostic genetic testing is most clinically useful where a patient has typical symptoms and signs (including motor abnormalities) of HD but has no known family history. However, it is also the situation where the diagnosis-once the implications have been fully absorbed-may cause the most distress for both immediate and extended family. HD will not be the only differential diagnosis. Where HD seems possible, the neurologist may suggest proceeding with genetic testing. It is best to inform both the patient and immediate relatives about the hereditary nature of the disease before carrying out the test. If the diagnosis is confirmed, the clinician must recognise the needs of the entire family as well as the index patient and ensure that family members can access appropriate genetic counselling services.

The patient with a positive family history and prodromal symptoms, which suggest the impending onset of HD

The current diagnostic criteria for HD still require motor abnormalities such as choreiform movements. Although chorea can sometimes be the presenting symptom, many patients with HD experience prodromal behavioural changes such as irritability, anxiety or depression before motor onset, ${ }^{13}{ }^{14}$ while others present with cognitive changes, often manifesting as difficulties in the workplace. Sometimes, these prodromal changes continue for a decade or more before the early motor signs appear. An unfavourable genetic test result in such circumstances does not exclude the possibility of a concurrent psychiatric disorder that is essentially unrelated to $\mathrm{HD}$ and thus cannot confirm the diagnosis. In such circumstances, it is preferable to adopt a tailored approach according to the patient's symptoms and key concerns. If the 
patient is not asking about his or her genetic status, it is appropriate to treat the presenting symptoms, for example, with selective serotonin-reuptake inhibitors for an existing depressive episode and arrange follow-up. Alternatively, if the patient finds it difficult to manage the uncertainty of not knowing his or her genetic status, as well as dealing with any symptoms, it would be appropriate to refer to the nearest genetic unit.

A major affective disorder or a psychotic condition such as schizophrenia appears more common in people who carry the HD gene mutation than in the general population; both conditions occur more frequently in some HD families than in others. ${ }^{15} 16$ This suggests that the HD mutation may exacerbate the risk of depressive disorder or schizophrenia in those people carrying an increased genetic susceptibility to depression or psychosis, by virtue of a distinct family history of one of these conditions. The increased risk of depression or psychosis occurs not only in the immediate prodrome of $\mathrm{HD}$, but may occur decades before the onset of motor symptoms. Again, an unfavourable genetic test result in this instance does not exclude a concurrent psychiatric disorder essentially unrelated to $\mathrm{HD}$ and thus cannot be regarded as confirming the diagnosis. Diagnostic genetic testing for HD is inappropriate in such circumstances and patients requesting this should be referred for predictive testing. Genetic units usually work in close collaboration with colleagues in psychiatry in such circumstances.

While the principles outlined above usually work well, there are occasional situations where a patient investigated for cognitive impairment presents with a neuropsychological test profile entirely consistent with HD, but has only non-specific motor changes insufficient to justify a confident clinical diagnosis of HD. In such a case, where HD seems the most likely diagnosis, genetic testing is entirely reasonable.

\section{The child with a family history of HD and features of juvenile HD}

Juvenile HD (onset $\leq 20$ years) poses particular diagnostic challenges. The phenotype may differ from the typical adult onset in that there may be more prominent poverty of movement and speech disturbance, especially in those rare cases with onset $\leq 10$ years. Criteria proposed by Nance for diagnostic testing in those with onset $\leq 10$ years are: ${ }^{17}$

- a known family history of HD (often, but not exclusively, the father)

and two or more of

- declining school performance

- seizures

- oral motor dysfunction

- rigidity

- gait disturbance.

Most juvenile HD cases, however, present in the second decade, with a similar clinical picture, but the typical 'adult onset' presentation is also possible. As with adult cases, there is no issue in arranging a genetic test if the child or young person has unequivocal neurological signs and a known family history of HD. There can be problems if the family history is unknown and, in exceptional cases, if a child has signs before the parent. However, the insidious onset of HD means that the parents are usually aware of nonspecific changes in their child ${ }^{1819}$ and may have concerns about school performance or behaviour before motor onset, which may or may not turn out to be HD related. It is crucial to support the parents and provide an individualised plan for follow-up, for example, arranging a baseline neuropsychological assessment ${ }^{2021}$ and possibly an MRI scan. ${ }^{21}$ Neither is diagnostic in itself, but may be repeated at a future time; the planned follow-up may reassure the parents that their concerns are being taken seriously and the clinician can judge whether the symptoms are progressing.

\section{Checklist before diagnostic genetic testing}

- Has the test been requested by a neurologist (or neuropsychiatrist) with relevant experience?

- Has the patient been appropriately counselled about the implications of genetic testing and possible test outcomes?

- Has the neurological examination shown evidence of motor symptoms?

- Have the patient's family or caregiver been included in discussions about the diagnostic genetic test and hereditary implications of $\mathrm{HD}$ ?

- Has the family been informed about the option of referral for genetic counselling?

- Has appropriate informed consent for diagnostic genetic testing been obtained by the patient/legal representative/person with parental responsibility?

- Is the laboratory to which the sample is being sent accredited and do they have experience of performing HD genetic testing?

- Have full clinical details, contact details of the requesting clinician and evidence that consent has been obtained noted on the laboratory request card?

- Has the timescale for expecting a result been made clear to the patient family and the laboratory?

- Has a face-to-face appointment with the person requesting the genetic test been arranged to give the result?

Checklist following a genetic diagnosis of HD

- Has a plan for follow-up been discussed with the patient and his/her family?

- Is there opportunity to refer the patient to a specialist HD management clinic?

- Have details of the lay organisation been provided?

- Have options to participate in research been discussed?

- Do the patient's relatives wish to be referred for genetic counselling? 
Clinicians should always offer follow-up to the patient and his or her family and should consider referring to a specialist HD management clinic. The opportunity to participate in research such as the REGISTRY or Enroll-HD studies ${ }^{22}$ provide the patient with a sense of purpose and may also offer future opportunities to participate in clinical trials. Family members may want to access genetic counselling, and the neurologist should be aware of the nearest genetic counselling unit and be able to provide details of the lay organisation for support. We suggest that a multidisciplinary approach to HD care-taking account of the needs and expectations of both the affected individual and their family-will meet with the greatest success.

\section{ONLINE RESOURCES}

- International Huntington Association http://www.huntington-assoc.com

- European Huntington's Disease Network http://www.euro-hd.net

- HD Buzz; http://www.hdbuzz.net

- Huntington Disease Youth Organisation http://www.hdyo.org

\section{Author affiliations}

${ }^{1}$ Faculty of Medicine and Human Sciences, Institute of Human Development, University of Manchester and Manchester Academic Health Science Centre, Manchester, UK

${ }^{2}$ Manchester Centre for Genomic Medicine, Central Manchester University Hospitals NHS Foundation Trust, Manchester, UK

${ }^{3}$ Instituto di Farmacologia Traslazionale del CNR, Rome, Italy

${ }^{4}$ Department of Clinical Genetics, Sheffield Children's Hospital, Sheffield, UK

${ }^{5}$ Centre for Human and Clinical Genetics, Leiden University Medical Centre (LUMC), Leiden, The Netherlands

${ }^{6}$ Neurogenetics Unit, Institute of Neurology, London, UK

${ }^{7}$ Memory Disorders Research Group, Section of Neurogenetics, Department of Neurology, Rigshospitalet, Copenhagen University Hospital, Copenhagen

${ }^{8}$ Department of Cellular and Molecular Medicine, Section of Neurogenetics, Panum Institute, University of Copenhagen, Copenhagen, Denmark

${ }^{9}$ NE Thames Regional Genetics Service, Great Ormond Street Hospital, London, UK ${ }^{10}$ Dipartimento di Neuroscienze, Riabilitazione, Oftalmologia, Genetica e Scienze Materno-Infantili
(DINOGMI), University of Genoa, Genoa, Italy

${ }^{11}$ UO of Medical Genetics, IRCCS AOU San Martino of Genova, Genoa, Italy

${ }^{12}$ Neurological Department, Fundación Jiménez Díaz, Madrid, Spain

${ }^{13}$ Department of Neurology, Leiden University Medical Centre (LUMC), Leiden, The Netherlands

Acknowledgements The authors are grateful to the European Huntington's Disease Network (EHDN), which is supported by the CHDI Foundation, a not-for-profit organisation dedicated to finding treatments for Huntington's disease. DC and RM also wish to acknowledge the support of the National Institute for Health Research (NIHR) Biomedical Research Centre in Manchester.

Contributors The original draft was written by Dr DC, Dr RM and Dr OQ following an outline suggested by Dr MF at a meeting of the Working Group. All the other authors were involved in critical review and editing of the manuscript.

Competing interests None.

Provenance and peer review Commissioned; externally peer reviewed. This paper was reviewed by Anne Rosser, Cardiff, UK.

\section{REFERENCES}

1 Kremer B. Clinical neurology of Huntington's disease. In: Bates GP, Harper PS, Jones L. eds. Huntington disease. 3rd edn. New York: Oxford University Press, 2002;28-61.

2 The Huntington's Disease Collaborative Research Group. A novel gene containing a trinucleotide repeat that is expanded and unstable on Huntington's disease chromosome. Cell 1993;72:971-83.

3 International Huntington Association and the World Federation of Neurology Research Group on Huntington's Chorea. Guidelines for the molecular genetics predictive test in Huntington's disease. J Med Genet 1994;31:555-9.

4 MacLeod R, Tibben A, Frontali M, et al. Recommendations for the predictive genetic test in Huntington's disease. Clin Genet 2013;83:221-3.

5 Paulsen JS, Hoth KF, Nehl C, et al. Critical periods of suicide risk in Huntington's disease. Am J Psychiatry 2005;162:725-31.

6 Hubers AAM, Reedeker N, Giltay EJ, et al. Suicidality in Huntington's disease. J Affect Disord 2012;136:550-7.

7 Snowden JS, Craufurd D, Griffiths HL, et al. Awareness of involuntary movements in Huntington's disease. Arch Neurol 1998;55:801-5.

8 Duff K, Paulsen JS, Beglinger LJ, et al. 'Frontal' behaviors before the diagnosis of Huntington's disease and their relationship to markers of disease progression: evidence of early lack of awareness. J Neuropsychiatry Clin Neurosci 2010;22:196-207.

9 Caine ED, Shoulson I. Psychiatric syndromes in Huntington's disease. Am J Psychiatry 1983;140:728-33.

10 Deckel AW, Morrison D. Evidence of a neurologically based "Denial of illness" in patients with Huntington's disease. Arch Clin Neuropsychol 1996;11:295-302.

11 Ho AK, Robbins AOG, Barker RA. Huntington's disease patients have selective problems with insight. Mov Disord 2006;21:385-9.

12 Hoth KF, Paulsen JS, Moser DJ, et al. Patients with Huntington's disease have impaired awareness of cognitive, emotional and functional abilities. J Clin Exp Neuropsychol 2007;29:365-76. 
13 Julien CL, Thompson JC, Wild S, et al. Psychiatric disorders in preclinical Huntington's disease. J Neurol Neurosurg Psychiatry 2007;78:939-43.

14 Loy CT, McCusker EA. Is a motor criterion essential for the diagnosis of Clinical Huntington Disease? PLoS Curr 2013 Apr 11;5. pii: ecurrents.hd. f4c66bd51e8db11f55e1701af937a419 doi:10.1371/currents. hd.f4c66bd51e8db11f55e1701af937a419

15 Folstein SE, Abbott MH, Chase GA, et al. The association of affective disorder with Huntington's disease in a case series and in families. Psychol Med 1983;13:537-42.

16 Tsuang D, DiGiacomo L, Lipe H, et al. Familial aggregation of Schizophrenia-like symptoms in Huntington's disease. Am J Med Genet 1998;81:323-7.

17 Nance MA, et al. Genetic testing of children at risk for Huntington's disease. Neurology 1997;49:1048-53.

18 Smith JA, Brewer HM, Eatough V, et al. The personal experience of juvenile Huntington's disease: an interpretative phenomenological analysis of parents' accounts of the primary features of a rare genetic condition. Clin Genet 2006;69:486-96.

19 Eatough V, Santini H, Eiser C, et al. The personal experience of parenting a child with juvenile Huntington's disease: perceptions across Europe. Eur J Hum Genet 2013;21: 1042-8.

20 Lehman RK, Nance M. Family history in juvenile Huntington disease. Do the signs point to "yes" or "very doubtful"? Neurology 2013;80:976-7.

21 Quarrell OW, Nance MA, Nopoulos P, et al. Managing juvenile Huntington's disease. Neurodegener Dis Manag 2013;3:267-76.

22 Orth M, Handley OJ, Schwenke C, et al. Investigators of the European HD Network. Observing Huntington's Disease: the European Huntington's Disease Network's REGISTRY. PLoS Curr 2010 Sep 28 [revised 2011 Apr 13];2. pii: RRN1184. 